Revista de MATEMÁtica: TeORÍA y APliCACIONEs 2017 24(2) : 201-225

CIMPA - UCR ISSN: 1409-2433 (PRINT), 2215-3373 (ONLINE)

\title{
MATEMÁTICA DIFUSA Y COMPLEJOS CÚBICOS
}

\author{
FUZZY MATHEMATICS AND CUBICAL \\ COMPLEXES
}

\author{
Adolfo MACEDA-MÉNDEZ*
}

Received: 13/Sep/2016; Revised: 18/Apr/2017;

Accepted: 28/Apr/2017

\begin{abstract}
Revista de Matemática: Teoría y Aplicaciones is licensed under a Creative Commons Reconocimiento-NoComercial-Compartirigual 4.0 International License.

Creado a partir de la obra en http://www.revistas.ucr.ac.cr/index.php/matematica

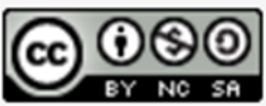

*Instituto de Física y Matemáticas, Universidad Tecnológica de la Mixteca, Oaxaca, México. E-Mail: amaceda901@hotmail.com
\end{abstract}




\title{
Resumen
}

La matemática difusa generaliza los conceptos tradicionales de la matemática utilizando los llamados conjuntos difusos, lo que permite modelar y estudiar de manera más apropiada fenómenos caracterizados por su imprecisión. Estas generalizaciones incluyen conceptos de álgebra, análisis y topología, entre otros. Por otra parte, los complejos cúbicos tienen aplicaciones al procesamiento de imágenes digitales y al estudio de los sistemas dinámicos, pero en la literatura actual no hay una extensión de sus propiedades utilizando conjuntos difusos. En este documento se propone una generalización del concepto de complejo cúbico y de algunas de sus características, tales como realización poliédrica, conexidad, componente conexa y agujero, utilizando conjuntos difusos. Se definen los árboles superior e inferior de un complejo cúbico difuso, los cuales proporcionan información sobre la manera en la que están relacionados sus extremos regionales. También se definen los grupos de homología de estos complejos cúbicos difusos y se demuestra que el rango del 0-grupo de homología de un nivel dado es igual al número de máximos regionales de dicho nivel. Por último, se muestra cómo se le puede asociar un complejo cúbico difuso a una imagen digital bidimensional en tonos de gris para estudiar algunas de sus propiedades topológicas.

Palabras clave: complejo cúbico; topología difusa; conexidad.

\begin{abstract}
Fuzzy mathematics generalize concepts of traditional mathematics using fuzzy sets. This enables to study and model more properly phenomens characterized by imprecision. These generalizations includes concepts of algebra, analysis and topology. On the other side, cubical complexes have applications in digital image processing and in the study of dynamical systems, but in the actual literature there is not an extension of their properties using fuzzy sets. In this paper is proposed a generalization of the concept of cubical complex and of some of their properties, such as connectedness, polyhedral realization, connected component and holes, using fuzzy sets. The upper and lower trees of a fuzzy cubical complex are defined, which give information about the way in which its regional extrema are related. The homology groups of a fuzzy cubical complex are defined and it is shown that the rank of the 0-homology group of a given level is equal with the number of regional maxima of that level. Finally, it is shown how to associate a fuzzy cubical complex with a bidimensional digital grayscale image in order to study somo of its topological properties.
\end{abstract}

Keywords: cubical complex; fuzzy topology; connectedness.

Mathematics Subject Classification: 54A40, $03 E 72$. 


\section{Introducción}

El concepto de conjunto difuso, introducido por Zadeh en [30], ha sido utilizado para extender diversos conceptos de la matemática tradicional, tales como algunos tipos de conjuntos (afines y convexos [14], acotados [30], conexos [12, 15]), estructuras algebraicas (subgrupos [22, 27], espacios vectoriales [16]) y estructuras discretas (grafos [31]). Una descripción más amplia de este tipo de extensiones puede encontrarse en [10]. Una generalización a los espacios topológicos fue propuesta por Chang en [6], mientras que Ying y Mao en [29] presentan un estudio muy amplio de los diversos conceptos de topología que se han extendido mediante conjuntos difusos. En el caso de la topología algebraica, se han propuesto generalizaciones del concepto de homotopía, como en [8], mientras que los grupos de homología, utilizando conjuntos difusos se definen, con diferentes enfoques, en $[28,26,2]$. En estos trabajos se presentan diversas propiedades de los grupos de homología relacionadas con algún tipo de homotopía, pero no se relaciona el rango de los grupos de homología difusa con conceptos de conexidad. Una forma de estudiar los grupos de homología para algunas aplicaciones en áreas como el procesamiento de imágenes, el reconocimiento de patrones y en el estudio de sistemas dinámicos lineales y no lineales, es mediante los llamados complejos cúbicos cerrados [9, 32, 20].

En este documento se presenta una generalización del concepto de complejo cúbico utilizando conjuntos difusos. Para ello, se proporciona una definición de complejo cúbico que incluye a los complejos cerrados y a los abiertos. Posteriormente se presentan la definición y propiedades básicas de los complejos cúbicos difusos, incluyendo algunas nociones de conexidad y su relación con los llamados extremos regionales. A partir de los conjuntos de nivel de un complejo cúbico difuso, se definen dos grafos que contienen información sobre los extremos regionales y se demuestra que estos grafos son árboles, a los cuáles se les puede dar una orientación canónica eligiendo un vértice apropiado. También se propone una definición de los grupos de homología de un complejo cúbico difuso, así como de sus números de Betti.

Entre los principales resultados se muestra que los máximos regionales tienen propiedades similares a las de las componentes conexas de un complejo cúbico cerrado, mientras que los mínimos regionales generalizan el concepto de agujero. Se demuestra que el 0-número de Betti de un nivel dado es igual al número de máximos regionales de dicho nivel del complejo cúbico difuso. También se explica cómo construir un complejo cúbico difuso a partir de una imagen digital bidimensional en tonos de gris y se demuestran algunos teoremas que establecen relaciones estrechas entre las propiedades topológicas de la imagen y las del complejo cúbico difuso correspondiente. 


\section{Complejos cúbicos}

En esta sección se recuerdan algunos conceptos que serán necesarios más adelante. Sea $\mathbb{R}^{n}$ el espacio euclidiano $n$-dimensional y $\mathbb{Z}$ el conjunto de números enteros. Una $k$-celda o celda $k$-dimensional en $\mathbb{R}^{n}$ es un conjunto de la forma

$$
s\left(x_{1}, \ldots, x_{n} ; \Lambda\right)=I_{1} \times I_{2} \times \ldots \times I_{n}
$$

donde $x_{1}, x_{2}, \ldots, x_{n} \in \mathbb{Z}, \Lambda$ es un subconjunto de $\{1,2, \ldots, n\}$ con $k$ elementos, $I_{\lambda}=\left\{x_{\lambda}+1 / 2\right\}$ si $\lambda \notin \Lambda$ e $I_{\lambda}=\left\{x_{\lambda}-1 / 2, x_{\lambda}+1 / 2\right\}$ si $\lambda \in \Lambda$. Los $2^{k}$ elementos de $\mathbb{R}^{n}$ que la forman son sus vértices. Cuando no sea relevante indicar los elementos $x_{1}, \ldots, x_{n}$, se escribirá simplemente $s$. La realización poliédrica de esta celda es

$$
\left|s\left(x_{1}, \ldots, x_{n} ; \Lambda\right)\right|=J_{1} \times J_{2} \times \ldots \times J_{n}
$$

donde $J_{\lambda}=\left\{x_{\lambda}+1 / 2\right\}$ si $\lambda \notin \Lambda$ y $J_{\lambda}=\left(x_{\lambda}-1 / 2, x_{\lambda}+1 / 2\right)$ si $\lambda \in \Lambda$. Nótese que esta realización poliédrica está definida como el producto cartesiano de $k$ intervalos abiertos y de $n-k$ puntos. Cuando $k=n$, esta celda es un conjunto abierto en $\mathbb{R}^{n}$ respecto a la topología usual. El conjunto de las realizaciones poliédricas de todas las celdas constituye una partición de $\mathbb{R}^{n}$. Una celda $s$ es cara de una celda $s^{\prime}$ si $s \subset s^{\prime}$. Si además $s \neq s^{\prime}$, se dice que $s$ es una cara propia de $s^{\prime}$. En este último caso las realizaciones poliédricas de $s$ y $s^{\prime}$ no tienen puntos en común, pero $|s|$ está contenida en la adherencia de $\left|s^{\prime}\right|$ y $|s| \cup\left|s^{\prime}\right|$ es un conjunto convexo.

Un complejo cúbico es cualquier colección de celdas. En particular, el conjunto de todas las $k$-celdas, con $k=0,1, \ldots, n$, es un complejo cúbico que se denota por $\left|\mathbb{Z}^{n}\right|$. Los elementos de

$$
\left\{\left(x_{1}+1 / 2, \ldots, x_{n}+1 / 2\right): x_{1}, \ldots, x_{n} \in \mathbb{Z}\right\}
$$

son los vértices de $\left|\mathbb{Z}^{n}\right|$. De acuerdo con esta definición, puede ocurrir que una cara de alguna celda de un complejo cúbico no pertenezca al complejo cúbico.

Dado un complejo cúbico $\mathbb{K}$, su complemento es el conjunto de las celdas de $\left|\mathbb{Z}^{n}\right|$ que no pertenecen a $\mathbb{K}$ y se denota por $\mathbb{K}^{c}$. La adherencia o cerradura del complejo cúbico $\mathbb{K}$ es el conjunto de las celdas que son cara de alguna celda de $\mathbb{K}$ y se denota por $\overline{\mathbb{K}}$. Esto define un operador de cerradura en el conjunto de los complejos cúbicos y por lo tanto induce una topología en él. Respecto a esta topología, un complejo cúbico $\mathbb{K}$ es cerrado si contiene todas las caras de sus celdas, y es abierto si su complemento es cerrado. Un complejo cúbico $\mathbb{L}$ es un subcomplejo de $\mathbb{K}$ si toda celda de $\mathbb{L}$ pertenece a $\mathbb{K}$. La realización poliédrica de 
$\mathbb{K}$, denotada por $|\mathbb{K}|$, es la unión de las realizaciones poliédricas de sus celdas. Esta realización es un subconjunto cerrado (abierto) de $\mathbb{R}^{n}$ con la topología usual si, y sólo si, el complejo cúbico es cerrado (abierto).

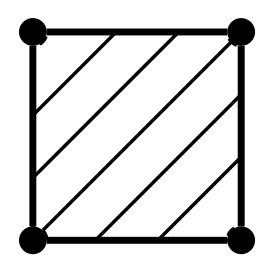

(a)

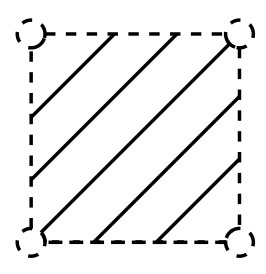

(b)

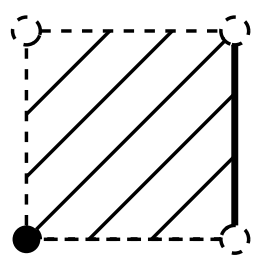

(c)

Figura 1: Realización poliédrica de complejos cúbicos.

En la Figura 1a se muestra la realización poliédrica de un complejo cúbico cerrado, formado por cuatro 0-celdas, cuatro 1-celdas y una 2-celda. La Figura $1 \mathrm{~b}$ muestra la realización poliédrica de un complejo cúbico abierto formado únicamente por una 2-celda, mientras que en la Figura 1c se muestra la realización poliédrica de un complejo cúbico que no es ni abierto ni cerrado, formado por una 0 -celda, una 1-celda y una 2-celda.

Dos celdas $s$ y $s^{\prime}$ de un complejo cúbico $\mathbb{K}$ están en relación de conexidad si existe una sucesión finita de celdas de $\mathbb{K}$, digamos $s_{0}=s, \ldots, s_{m}=s^{\prime}$, tal que $s_{i}$ es cara de $s_{i+1}$ o $s_{i+1}$ es cara de $s_{i}$ para todo $i=0,1, \ldots, m-1$. Esto define una relación de equivalencia en $\mathbb{K}$. Sus clases de equivalencia son subcomplejos de $\mathbb{K}$ llamados componentes conexas de $\mathbb{K}$. El complejo cúbico $\mathbb{K}$ es conexo si tiene una única componente conexa. Las componentes conexas de la realización poliédrica del complejo cúbico $\mathbb{K}$ son las realizaciones poliédricas de las componentes conexas de $\mathbb{K}$.

\section{Conjuntos difusos}

A partir de esta sección, $I$ denotará al intervalo unitario $[0,1]$.

Dado un conjunto ordinario $X$, un conjunto difuso en $X$ es una función $A: X \rightarrow I$. Al valor de $A$ en un elemento $x \in X$ se le llama el grado de pertenencia de $x$ al conjunto difuso $A$. Cualquier subconjunto ordinario de $X$ se puede ver como un conjunto difuso en $X$ mediante su función característica. Dado $a \in I$, se define $\underline{a}$ como el conjunto difuso en $X$ que tiene el valor constante $a$. 
Con $I^{X}$ se denotará a la familia de todos los conjuntos difusos en $X$. En este conjunto se pueden definir operaciones de unión e intersección como sigue: $\mathrm{Si}$ para $\lambda \in \Lambda, A_{\lambda}: X \rightarrow I$ es un conjunto difuso en $X$ :

$$
\begin{aligned}
& \left(\bigvee_{\lambda \in \Lambda} A_{\lambda}\right)(x)=\sup _{\lambda \in \Lambda}\left\{A_{\lambda}(x)\right\} \ldots \text { unión, } \\
& \left(\bigwedge_{\lambda \in \Lambda} A_{\lambda}\right)(x)=\inf _{\lambda \in \Lambda}\left\{A_{\lambda}(x)\right\} \ldots \text { intersección. }
\end{aligned}
$$

El complemento de un conjunto difuso $A$ está dado por

$$
A^{c}(x)=1-A(x)
$$

Un conjunto difuso $A$ es un subconjunto del conjunto difuso $B$ si

$$
A(x) \leq B(x) \text { para todo } x \in X
$$

En este caso se escribe $A \leq B$.

Estas definiciones generalizan las usuales si se consideran las funciones características. Además, estas operaciones son conmutativas, asociativas, distributivas y satisfacen las leyes de De Morgan. No se cumple, en general, que $A \vee A^{c}=\underline{1}$ о $A \wedge A^{c}=\underline{0}$.

Dado $A \in I^{X}$ y $a \in I$, se define el $a$-nivel superior cerrado de $A$ como el conjunto ordinario $A_{[a]}=\{x \in X: A(x) \geq a\}$. De manera similar se denotan

$$
\begin{aligned}
& A_{(a)}=\{x \in X: A(x)>a\}, \\
& A^{[a]}=\{x \in X: A(x) \leq a\}, \\
& A^{(a)}=\{x \in X: A(x)<a\} .
\end{aligned}
$$

El soporte del conjunto difuso $A$ es

$$
\operatorname{supp}(A)=\{x \in X: A(x) \neq 0\}
$$

mientras que su rango es

$$
\operatorname{rank}(A)=\{a \in I: a=A(x) \text { para algún } x \in X\} .
$$




\section{Topología difusa}

A una colección $\tau \subset I^{X}$ que satisface las condiciones:

1. $\underline{0}, \underline{1} \in \tau$;

2. $A, B \in \tau$ implica $A \wedge B \in \tau$;

3. $A_{\lambda} \in \tau, \lambda \in \Lambda$ implica $\bigvee_{\lambda \in \Lambda} A_{\lambda} \in \tau$;

se le llama una topología difusa en $X$ y $(X, \tau)$ es un espacio topológico difuso. Los elementos de $\tau$ son los conjuntos abiertos. Un conjunto difuso en $X$ es cerrado si su complemento es abierto.

A partir de un espacio topológico ordinario $(X, T)$ se puede construir una topología difusa en $X$ considerando las funciones semicontinuas inferiormente. Una función $f: X \rightarrow I$ es semicontinua inferiormente si $f^{[a]}=\{x \in X$ : $f(x) \leq a\}$ es un conjunto cerrado en $X$, para todo $a \in I$. La colección de las funciones de $X$ en $I$ que son semicontinuas inferiormente constituye una topología difusa en $X$ que se denota por $\omega(X)$. En este caso, la función característica de cualquier conjunto abierto es un elemento de esta topología difusa. Además, un conjunto difuso $A$ en $X$ es abierto si, y sólo si, cada conjunto $A^{[a]}$ es cerrado. Equivalentemente, cada $A_{(a)}$ debe ser un conjunto abierto para que $A$ sea un conjunto abierto. Caracterizaciones similares se tienen para los conjuntos cerrados respecto a esta topología.

En la topología difusa es importante extender propiedades topológicas, tales como la conexidad y la compacidad, para que se puedan estudiar en un espacio topológico difuso. Sin embargo, esto se puede hacer de diversas maneras. Por ejemplo, para la propiedad de conexidad, en [15] se presentan dos formas distintas de extenderla, mientras que en [13] se comparan varias extensiones de la propiedad de compacidad. A fin de determinar si estas extensiones son buenas, Lowen propone que una propiedad $P^{\prime}$ para los espacios topológicos difusos es una buena extensión de una propiedad $P$ para los espacios topológicos si, y sólo si, cada vez que el espacio topológico $(X, T)$ tiene la propiedad $P$, el espacio topológico difuso $(X, \omega(T))$ tiene la propiedad $P^{\prime}$.

Una discusión más extensa sobre conjuntos difusos y topología difusa puede consultarse en [29].

\section{Complejos cúbicos difusos}

Una celda difusa se define como un conjunto difuso cuyas propiedades se relacionan con las de las celdas ordinarias definidas anteriormente. 
Definición 1 Una $k$-celda difusa es un conjunto difuso s en $\mathbb{R}^{n}$ cuyo soporte es una $k$-celda y que es constante en dicho soporte. El valor de $k$ es la dimensión de la celda difusa y su valor constante es el grado de la celda, denotado por $\operatorname{deg}(s)$. Los elementos de su soporte son sus vértices. El conjunto de todas las celdas difusas se denota por $\left|\mathbb{Z}^{n}\right|_{I}$.

A partir de estas celdas difusas se definen los complejos cúbicos difusos.

Definición 2 Un complejo cúbico difuso $\mathbb{K}$ es cualquier colección de elementos de $\left|\mathbb{Z}^{n}\right|_{I}$ tal que celdas difusas diferentes tienen distintos soportes.

La última condición de esta definición implica que si $s$ y $s^{\prime}$ son celdas difusas distintas del complejo cúbico difuso $\mathbb{K}$ entonces las realizaciones poliédricas de sus soportes no tienen puntos en común.

Para los complejos cúbicos difusos se definen su soporte y sus secciones de forma similar al caso de los conjuntos difusos.

Definición 3 Dado un complejo cúbico difuso $\mathbb{K}$, su soporte es el complejo cúbico ordinario definido por

$$
\operatorname{supp}(\mathbb{K})=\{\operatorname{supp}(s): s \in \mathbb{K}\}
$$

y su fondo, denotado por $\mathrm{Bk}(\mathbb{K})$ es el complejo complemento de su soporte.

Definición 4 Si $\mathbb{K}$ es un complejo cúbico difuso, para cada a $\in I-\{0\}$, su a-nivel $\mathbb{K}_{[a]}$ es la colección de los soportes de sus celdas de grado $\geq a$. El conjunto $\mathbb{K}_{[0]}$ es, por definición, el complejo cúbico $\left|\mathbb{Z}^{n}\right|$. De manera similar, para $a \neq 0$ se define

$$
\mathbb{K}^{(a)}=\{\operatorname{supp}(s): s \in \mathbb{K}, \operatorname{deg}(s)<a\} \cup \operatorname{Bk}(\mathbb{K})
$$

mientras que $\mathbb{K}^{(0)}$ es el complejo cúbico vacío. Para todo a $\in$ I se definen

$$
\begin{aligned}
\mathbb{K}_{(a)} & =\{\operatorname{supp}(s): s \in \mathbb{K}, \operatorname{deg}(s)>a\} \\
\mathbb{K}^{[a]} & =\{\operatorname{supp}(s): s \in \mathbb{K}, \operatorname{deg}(s) \leq a\} \cup \operatorname{Bk}(\mathbb{K})
\end{aligned}
$$

Todos estos conjuntos son complejos cúbicos ordinarios, $\mathbb{K}_{(0)}=\operatorname{supp}(\mathbb{K})$, $\mathbb{K}^{[0]}=\operatorname{Bk}(\mathbb{K}), \mathbb{K}_{(1)}=\emptyset$ y $\mathbb{K}^{[1]}=\left|\mathbb{Z}^{n}\right|$.

Los complejos cúbicos difusos se clasifican en cerrados o abiertos, dependiendo de las propiedades de sus conjuntos de nivel, de forma similar a la caracterización de los conjuntos abiertos o cerrados respecto a la topología difusa de las funciones semicontinuas inferiormente. 
Definición 5 Un complejo cúbico difuso $\mathbb{K}$ es:

1. Cerrado si $\mathbb{K}_{[a]}$ es un complejo cúbico cerrado para todo a $\in I$.

2. Abierto si $\mathbb{K}^{[a]}$ es un complejo cúbico cerrado para cada $a \in I$.

Puesto que $\mathbb{K}_{[a]}=\left(\mathbb{K}^{(a)}\right)^{c}$ y $\mathbb{K}^{[a]}=\left(\mathbb{K}_{(a)}\right)^{c}$, es claro que un complejo cúbico difuso $\mathbb{K}$ es:

1. Cerrado si, y sólo si, $\mathbb{K}^{(a)}$ es un complejo cúbico abierto para cada $a \in I$.

2. Abierto si, y sólo si, $\mathbb{K}_{(a)}$ es un complejo cúbico abierto para todo $a \in I$.

Para algunas discusiones posteriores, relacionadas con conceptos de conexidad, será útil la siguiente definición.

Definición 6 El rango de un complejo cúbico difuso $\mathbb{K}$ cuyo fondo es vacío, denotado por $\operatorname{rank}(\mathbb{K})$, es el conjunto de elementos $a \in I$ para los cuales existe una celda difusa $s \in \mathbb{K}$ tal que $\operatorname{deg}(s)=a$. Si el fondo de $\mathbb{K}$ es no vacío, se agrega el elemento 0 al conjunto anterior.

Puesto que el conjunto de celdas en $\mathbb{R}^{n}$ es numerable, se deduce que el rango de un complejo cúbico difuso es un subconjunto numerable de $I$.

\section{Realización poliédrica de complejos cúbicos difusos}

Definición 7 Si s es una celda difusa, su realización poliédrica es el conjunto difuso en $\mathbb{R}^{n},|s|: \mathbb{R}^{n} \rightarrow$ I cuyo soporte es la realización poliédrica del soporte de s y cuyo grado de pertenencia en los puntos de su soporte es $\operatorname{deg}(s)$. La realización poliédrica de un complejo cúbico difuso $\mathbb{K}$ se define como el conjunto difuso en $\mathbb{R}^{n}$ dado por

$$
|\mathbb{K}|=\bigvee_{s \in \mathbb{K}}|s|
$$

Una propiedad importante de la realización poliédrica de un complejo cúbico difuso está dada en el siguiente lema.

Lema 1 Sea $\mathbb{K}$ un complejo cúbico difuso y $x \in \mathbb{R}^{n}$. Si $|\mathbb{K}|(x)>0$, entonces existe una única celda difusa $s \in \mathbb{K}$ tal que $|\mathbb{K}|(x)=|s|(x)$. 
Demostración. Si $|\mathbb{K}|(x)>0$, entonces $\sup _{s \in \mathbb{K}}|s|(x)>0$ y por tanto existe una celda difusa $s \in \mathbb{K}$ tal que $|s|(x)>0$. Esto indica que $x$ pertenece al soporte de $|s|$ y por tanto $\left|s^{\prime}\right|(x)=0$ para cualquier otra celda difusa $s^{\prime} \in \mathbb{K}$. En consecuencia, $|\mathbb{K}|(x)=|s|(x)$.

Algunas relaciones entre los $a$-niveles de un complejo cúbico difuso y los de su realización poliédrica se presentan en el siguiente teorema.

Teorema 1 Sea $\mathbb{K}$ un complejo cúbico difuso. Para todo a $\in I$ :

1. $\left|\mathbb{K}_{[a]}\right|=|\mathbb{K}|_{[a]}$.

2. $\left|\mathbb{K}_{(a)}\right|=|\mathbb{K}|_{(a)}$.

3. $\left|\mathbb{K}^{[a]}\right|=\mid \mathbb{K}^{[a]}$.

4. $\left|\mathbb{K}^{(a)}\right|=|\mathbb{K}|^{(a)}$.

Demostración. Sólo se demostrará 1. Si $a \neq 0$, dado $x \in|\mathbb{K}|_{[a]}$, por el Lema 1 se cumple $|s|(x) \geq a$ para una única celda difusa $s \in \mathbb{K}$. Con esto, $x \in \operatorname{supp}(s)$ $\mathrm{y} \operatorname{deg}(s) \geq a$, de donde $\operatorname{supp}(s) \in \mathbb{K}_{[a]}$ y $x \in\left|\mathbb{K}_{[a]}\right|$.

Ahora, si $x \in\left|\mathbb{K}_{[a]}\right|$, entonces $x \in|s|$ para alguna celda $s \in \mathbb{K}_{[a]}$. Como $s=\operatorname{supp}\left(s^{\prime}\right)$ para alguna celda difusa $s^{\prime} \in \mathbb{K} \operatorname{con} \operatorname{deg}\left(s^{\prime}\right) \geq a$, entonces $|\mathbb{K}|(x)=\left|s^{\prime}\right|(x) \geq a$, con lo que $x \in|\mathbb{K}|_{[a]}$.

Cuando $a=0$, es clara la igualdad.

Utilizando el teorema 1 se obtiene la siguiente caracterización importante de los complejos cúbicos difusos cerrados (abiertos).

Teorema 2 Un complejo cúbico difuso es cerrado (abierto) si, y sólo si, su realización poliédrica es un conjunto difuso cerrado (abierto) en $\mathbb{R}^{n}$ con respecto a la topología difusa $\omega\left(\mathbb{R}^{n}\right)$.

\section{Conexidad de complejos cúbicos difusos}

En esta sección se presentan definiciones relacionadas con el concepto de conexidad para complejos cúbicos difusos y se demuestran algunas de sus propiedades.

La conexidad de un complejo cúbico difuso se define a partir de la conexidad de sus $a$-niveles superiores cerrados.

Definición 8 Un complejo cúbico difuso $\mathbb{K}$ es conexo si todo a-nivel superior cerrado $\mathbb{K}_{[a]}$ es un complejo cúbico conexo. 
En el caso de los complejos cúbicos difusos que no son conexos, el concepto de máximo regional tiene un papel similar al de componente conexa.

Definición 9 Sea $\mathbb{K}$ un complejo cúbico difuso. Un máximo regional de $\mathbb{K}$ de nivel $a>0$ es un complejo cúbico ordinario $\mathbb{L}$ que satisface:

1. $\mathbb{L}$ es una componente conexa del complejo cúbico $\mathbb{K}_{[a]}$.

2. Para todo $b>a, \mathbb{L} \cap \mathbb{K}_{[b]}=\emptyset$.

De manera análoga, los mínimos regionales tienen un papel similar al de las componentes conexas del complemento de un subconjunto del plano.

Definición 10 Un mínimo regional de nivel a, $0<a<1$, de $\mathbb{K}$ es un complejo cúbico ordinario $\mathbb{L}$ tal que

1. $\mathbb{L}$ es una componente conexa del complejo cúbico $\mathbb{K}^{[a]}$.

2. Para todo $b<a, \mathbb{L} \cap \mathbb{K}^{[b]}=\emptyset$.

Un mínimo regional de nivel 0 de $\mathbb{K}$ es una componente conexa de $\mathrm{Bk}(\mathbb{K})$.

Definición 11 Un extremo regional de nivel a de $\mathbb{K}$ es cualquier complejo cúbico ordinario que es un máximo o un mínimo regional de nivel a de $\mathbb{K}$.

Las celdas de un extremo regional de un complejo cúbico difuso están estrechamente relacionadas con las celdas difusas de dicho complejo cúbico, como se muestra en el siguiente teorema.

Teorema 3 Sea $\mathbb{K}$ un complejo cúbico difuso y $\mathbb{L}$ un complejo cúbico ordinario.

1. Si $\mathbb{L}$ es un máximo regional de $\mathbb{K}$ de nivel a, entonces, para cada celda $s \in \mathbb{L}$ existe una única celda difusa $s^{\prime} \in \mathbb{K}$ tal que $\operatorname{supp}\left(s^{\prime}\right)=s$ y $\operatorname{deg}\left(s^{\prime}\right)=a$.

2. Si $\mathbb{L}$ es un mínimo regional de $\mathbb{K}$ de nivel a, $0<a<1$, entonces, para cada celda $s \in \mathbb{L}$ existe una única celda difusa $s^{\prime} \in \mathbb{K}$ con $\operatorname{supp}\left(s^{\prime}\right)=s$ $y \operatorname{deg}\left(s^{\prime}\right)=a$.

Demostración. Sólo se probará 1 . Dado una celda $s \in \mathbb{L}$, existe una única celda difusa $s^{\prime} \in \mathbb{K}$ con $s=\operatorname{supp}\left(s^{\prime}\right)$ y $\operatorname{deg}\left(s^{\prime}\right) \geq a$. Si $\operatorname{deg}\left(s^{\prime}\right)>a$, definiendo $b=\operatorname{deg}\left(s^{\prime}\right)$ se tiene que $s \in \mathbb{L} \cap \mathbb{K}_{[b]}$ con $b>a$, contradiciendo la definición de máximo regional. Por tanto, $\operatorname{deg}\left(s^{\prime}\right)=a$.

Las componentes conexas de los $a$-niveles superiores cerrados de un complejo cúbico difuso tienen propiedades similares a las de las componentes conexas de un complejo cúbico ordinario. 
Lema 2 Si $\mathbb{K}$ es un complejo cúbico difuso y $\mathbb{L}_{1}$, $\mathbb{L}_{2}$ son componentes conexas de $\mathbb{K}_{\left[a_{1}\right]}$ y de $\mathbb{K}_{\left[a_{2}\right]}$, respectivamente, con $a_{1}>a_{2}$, entonces $\mathbb{L}_{1} \cap \mathbb{L}_{2}=\emptyset$ o $\mathbb{L}_{1} \subset \mathbb{L}_{2}$.

Demostración. Basta observar que $\mathbb{L}_{1}$ es un subcomplejo conexo de $\mathbb{K}_{\left[a_{2}\right]}$ y que $\mathbb{L}_{2}$ es una componente conexa de $\mathbb{K}_{\left[a_{2}\right]}$.

Teorema $4 \mathrm{Si} \mathbb{K}$ es un complejo cúbico difuso cerrado entonces sus máximos regionales son complejos cúbicos cerrados.

Demostración. Si $\mathbb{L}$ es un máximo regional de nivel $a$ de $\mathbb{K}$, entonces su realización poliédrica $|\mathbb{L}|$ es una componente conexa del conjunto cerrado $\left|\mathbb{K}_{[a]}\right|$ en $\mathbb{R}^{n}$ y por tanto es un conjunto cerrado. Así, $\mathbb{L}$ es un complejo cúbico cerrado.

Este resultado es similar al correspondiente para las componentes conexas de un conjunto cerrado en un espacio topológico ordinario.

En el caso de los mínimos regionales se tiene el siguiente resultado análogo.

Teorema 5 Los mínimos regionales de un complejo cúbico difuso abierto son complejos cúbicos cerrados.

El siguiente teorema muestra la relación entre conexidad y máximos regionales de un complejo cúbico difuso.

Teorema 6 Un complejo cúbico difuso $\mathbb{K}$ con rango finito es conexo si, y sólo si, tiene un único máximo regional.

Demostración. Sean $a_{1}<a_{2}<\ldots<a_{k}$ los elementos del rango de $\mathbb{K}$. Si $\mathbb{K}$ es conexo, entonces $\mathbb{K}_{\left[a_{k}\right]}$ es un máximo regional de nivel $a_{k}$. Además, para todo $a<a_{k}, \mathbb{K}_{[a]}$ es conexo y contiene a $\mathbb{K}_{\left[a_{k}\right]}$, por lo que no hay máximos regionales de nivel $a$ de $\mathbb{K}$.

Ahora, si $\mathbb{K}$ tiene un único máximo regional, como cualquier componente conexa de $\mathbb{K}_{\left[a_{k}\right]}$ es un máximo regional de nivel $a_{k}$ de $\mathbb{K}$, se deduce que $\mathbb{K}_{\left[a_{k}\right]}$ es conexo y corresponde al único máximo regional de $\mathbb{K}$. Además, una componente conexa $\mathbb{L}$ de $\mathbb{K}_{\left[a_{k-1}\right]}$ no es un máximo regional de $\mathbb{K}$, por lo que $\mathbb{L} \cap \mathbb{K}_{\left[a_{k}\right]} \neq \emptyset$ y $\mathbb{L} \cup \mathbb{K}_{\left[a_{k}\right]}$ es un complejo cúbico conexo contenido en $\mathbb{K}_{\left[a_{k-1}\right]}$ y por tanto $\mathbb{L}=$ $\mathbb{L} \cup \mathbb{K}_{\left[a_{k}\right]}$. Así, $\mathbb{K}_{\left[a_{k}\right]} \subset \mathbb{L}$. Se deduce que $\mathbb{K}_{\left[a_{k-1}\right]}$ tiene una única componente conexa y en consecuencia es conexo. El mismo razonamiento se puede aplicar a los complejos cúbicos $\mathbb{K}_{[a]}$ para los elementos restantes $a$ en el rango de $\mathbb{K}$. Por tanto, $\mathbb{K}$ es conexo.

El resultado de este teorema no se cumple sin la hipótesis de que el rango sea finito. 
Ejemplo 1 Considérese el complejo cúbico difuso $\mathbb{K}$ en $\mathbb{R}$, formado por

1. Las 0 -celdas cuyo soporte es $\{n+1 / 2\}$ y cuyo grado es $1-1 / n$.

2. Las 1 -celdas con soporte $\{n-1 / 2, n+1 / 2\}$ y grado $1-1 / n$,

para $n \in \mathbb{N}$ con $n \geq 2$.

El complejo cúbico $\mathbb{K}_{[a]}$ es conexo para todo a $\in I$, pero $\mathbb{K}$ no tiene máximos regionales.

Además, si se agrega la 0 -celda difusa con soporte $\{3 / 2\}$ y grado 1 , se obtiene un complejo cúbico difuso con un único máximo regional, pero en el que los complejos cúbicos $\mathbb{K}_{[a]}$ no son conexos.

Sea $\mathbb{K}$ un complejo cúbico difuso con rango finito y sean $a_{1}<\ldots<a_{k}$, sus elementos no nulos. Las componentes conexas de $\mathbb{K}_{\left[a_{k}\right]}$ son máximos regionales de nivel $a_{k}$ de $\mathbb{K}$. Además, si $l<k$, una componente conexa de $\mathbb{K}_{\left[a_{l}\right]}$ es un máximo regional de nivel $a_{l}$ de $\mathbb{K}$ si, y sólo si, $\mathbb{L} \cap \mathbb{K}_{\left[a_{l+1}\right]}=\emptyset$.

De forma similar, si el fondo de $\mathbb{K}$ es no vacío, definiendo $a_{0}=0$, las componentes conexas de $\mathbb{K}^{\left[a_{0}\right]}$ son mínimos regionales de $\mathbb{K}$ de nivel $a_{0}$. Para $k>l>0$, una componente conexa de $\mathbb{K}^{\left[a_{l}\right]}$ es un mínimo regional de $\mathbb{K}$ de nivel $a_{l}$ si, y sólo si, $\mathbb{L} \cap \mathbb{K}^{\left[a_{l-1}\right]}=\emptyset$.

Teorema 7 Sea $\mathbb{K}$ un complejo cúbico difuso con rango finito

$$
\operatorname{rank}(\mathbb{K})=\left\{a_{0}, \ldots, a_{k}\right\},
$$

donde $a_{0}<\ldots<a_{k}$. Si $\mathbb{L}$ es un mínimo regional de nivel $a_{i}$ de $\mathbb{K}$ para algún $i<k$ y $a_{i} \neq 0$, entonces es una componente conexa de $\left(\mathbb{K}_{\left[a_{i+1}\right]}\right)^{c}$.

Demostración. Basta observar que $\left(\mathbb{K}_{\left[a_{i+1}\right]}\right)^{c}=\mathbb{K}^{\left(a_{i+1}\right)}=\mathbb{K}^{\left[a_{i}\right]}$ si $i<k \mathrm{y}$ $a_{i} \neq 0$.

De forma similar se demuestra que si $\mathbb{L}$ es un máximo regional de nivel $a_{i}$ del complejo cúbico difuso $\mathbb{K}$, entonces es una componente conexa de $\left(\mathbb{K}^{\left[a_{i-1}\right]}\right)^{c}$.

Utilizando este resultado, se deducen las siguientes propiedades de los extremos regionales.

Corolario 1 Sea $\mathbb{K}$ un complejo cúbico difuso con rango finito.

1. Si $\mathbb{K}$ es cerrado, sus mínimos regionales son complejos cúbicos abiertos.

2. Si $\mathbb{K}$ es abierto, sus máximos regionales son complejos cúbicos abiertos. 


\section{8 Árboles de un complejo cúbico difuso}

Con el fin de estudiar la forma en la que se relacionan entre sí los extremos regionales de un complejo cúbico difuso, se pueden construir dos grafos a partir de dichos extremos, siguiendo la idea utilizada en [17].

Un grafo no dirigido es una pareja $G=(V, E)$ en la que $V$ es un conjunto no vacío cuyos elementos son los vértices del grafo y los elementos de $E$, llamados aristas, son conjuntos de la forma $\{u, v\}$ con $u, v \in V$ y $u \neq v$. Dos vértices $u, v$ son adyacentes si $\{u, v\} \in E$. El grafo $G$ es conexo si para cualesquiera dos vértices $u, v$ existe una sucesión de vértices $v_{0}=u, v_{1}, \ldots, v_{n}=v$ tales que $\left\{v_{i-1}, v_{i}\right\} \in E$ para $i=1,2, \ldots, n$. Un ciclo en $G$ es una sucesión de vértices $v_{0}, v_{1}, \ldots, v_{n}$ tal que $v_{0}=v_{n}$ y para cada $i=0, \ldots, n-2$ los vértices $v_{i}, v_{i+1}$ y $v_{i+2}$ son distintos entre sí. Este ciclo es simple si los vértices $v_{0}, v_{1}, \ldots, v_{n-1}$ son distintos. Un grafo conexo que no tiene ciclos simples es un árbol.

Sea $T=(V, E)$ un árbol en el que $V$ es finito y sea $r$ uno de sus vértices, llamado raíz. A cada vértice $v$ se le asigna un número natural $l(v)$, llamado su nivel, de la siguiente forma. La raíz $r$ tiene nivel 0. Cada vértice que sea adyacente a la raíz tiene nivel 1 . Dos vértices de nivel 1 no pueden ser adyacentes porque en ese caso se tendría un ciclo simple utilizando la raíz y estos vértices. A cada vértice que sea adyacente a un vértice de nivel 1 y que no sea la raíz se le asigna el nivel 2. Este proceso se puede continuar de forma inductiva. Así, cada arista del árbol tiene vértices $\{u, v\}$, y se puede suponer que $l(v)=l(u)+1$; el vértice $u$ es el inicio y el vértice $v$ es el final de la arista y se dice que $u$ es antecesor de $v$ o que $v$ es sucesor de $u$. De acuerdo con esto, cada vértice puede tener 0 o varios sucesores y cada vértice, excepto la raíz, tiene un único antecesor. A un vértice que no tiene sucesores se le llama una hoja. Con esto $(V, E, r)$ es un árbol dirigido.

Utilizando las componentes conexas de los $a$-niveles de un complejo cúbico difuso se pueden construir dos grafos.

Definición 12 Sea $\mathbb{K}$ un complejo cúbico difuso con soporte finito y con rango finito $\operatorname{rank}(\mathbb{K})=\left\{a_{0}=0, \ldots, a_{k}\right\}$. Se construye un grafo no dirigido, denotado por $T^{\mathbb{K}}$, de la siguiente manera. Los vértices del grafo son parejas ordenadas de la forma $(\mathbb{L}, i)$ donde $\mathbb{L}$ es una componente conexa del complejo $\mathbb{K}_{\left[a_{i}\right]}$. Sus aristas son los conjuntos $\left\{\left(\mathbb{L}_{1}, i\right),\left(\mathbb{L}_{2}, i+1\right)\right\}$ donde $\mathbb{L}_{1}$ es una componente de $\mathbb{K}_{\left[a_{i}\right]}, \mathbb{L}_{2}$ es una componente de $\mathbb{K}_{\left[a_{i+1}\right]} y \mathbb{L}_{2} \subset \mathbb{L}_{1}$, para $i \geq 0$.

Una propiedad importante de este grafo está dada en el siguiente teorema.

Teorema 8 El grafo $T^{\mathbb{K}}$ es un árbol. 
Demostración. Para demostrar que $T^{\mathbb{K}}$ es conexo, nótese que dado cualquier vértice $\left(\mathbb{L}_{0}, i+1\right)$ de $T^{\mathbb{K}}$, al ser $\mathbb{L}_{0}$ un complejo conexo contenido en $\mathbb{K}_{\left[a_{i}\right]}$, hay una componente conexa $\mathbb{L}_{1}$ de $\mathbb{K}_{\left[a_{i}\right]}$ que lo contiene. Por inducción, se obtiene una sucesión

$$
\left(\mathbb{L}_{0}, i+1\right),\left(\mathbb{L}_{1}, i\right), \ldots,\left(\mathbb{L}_{i+1}, 0\right)
$$

en la que vértices consecutivos forman una arista del grafo. Como $\mathbb{K}_{\left[a_{0}\right]}=\left|\mathbb{Z}^{n}\right|$, se deduce que el grafo es conexo.

Supóngase ahora que $\left(\mathbb{L}_{0}, i_{0}\right), \ldots,\left(\mathbb{L}_{n}, i_{n}\right)$ es un ciclo simple de $T^{\mathbb{K}}$. Hay dos posibilidades: $i_{1}=i_{0}+1$ o $i_{0}=i_{1}+1$. En el primer caso, $\mathbb{L}_{1} \subset \mathbb{L}_{0}$. Si $i_{1}=$ $i_{2}+1$ entonces $\mathbb{L}_{1} \subset \mathbb{L}_{2}$ y $\mathbb{L}_{2}$ es una componente de $\mathbb{K}_{\left[a_{i_{0}}\right]}$ cuya intersección con $\mathbb{L}_{1}$ es no vacía y por tanto $\mathbb{L}_{0}=\mathbb{L}_{2}$, lo que contradice la definición de ciclo. En consecuencia, $i_{2}=i_{1}+1$ y $\mathbb{L}_{2} \subset \mathbb{L}_{1}$. Por inducción, se deduce que

$$
\mathbb{L}_{0} \subset \mathbb{L}_{n-1} \subset \ldots \subset \mathbb{L}_{1} \subset \mathbb{L}_{0}
$$

lo que contradice la definición de ciclo.

Este árbol puede hacerse un árbol dirigido tomando como raíz $\mathbb{K}_{\left[a_{0}\right]}=\left|\mathbb{Z}^{n}\right|$. En este caso, el vértice $(\mathbb{L}, i)$ es una hoja si, y sólo si, $\mathbb{L}$ es un máximo regional de nivel $a_{i}$.

De forma similar se define un grafo $T_{\mathbb{K}}$ considerando las componentes de los complejos $\mathbb{K}^{\left[a_{i}\right]}$. Este grafo también es un árbol y, tomando como raíz a $\mathbb{K}^{\left[a_{k}\right]}=\left|\mathbb{Z}^{n}\right|$, los mínimos regionales corresponden a sus hojas.

\section{Grupos de homología}

A cada complejo cúbico difuso se le pueden asociar grupos de homología utilizando sus $a$-niveles, de tal manera que se relacione su rango con el número de máximos regionales.

Definición 13 Dado un complejo cúbico difuso $\mathbb{K}$ con soporte finito y rango $\operatorname{rank}(\mathbb{K})=\left\{a_{0}, \ldots, a_{k}\right\}$, su m-ésimo grupo de homología de nivel $a_{i}$ se define como

$$
H_{m}\left(\mathbb{K}, a_{i}\right)=H_{m}\left(\left|\mathbb{K}_{\left[a_{i}\right]}\right|,\left|\mathbb{K}_{\left[a_{i+1}\right]}\right|\right) .
$$

El rango de este grupo es el m-ésimo número de Betti de nivel $a_{i}$ y se denota por $\beta_{m}\left(\mathbb{K}, a_{i}\right)$.

En la definición anterior se consideran los grupos de homología singular de las realizaciones poliédricas de los $a$-niveles indicados. 
Puesto que el 0-número de Betti del par $\left.\left(\mid \mathbb{K}_{\left[a_{i}\right]}\right],\left|\mathbb{K}_{\left[a_{i+1}\right]}\right|\right)$ es igual al número de componentes de $\mathbb{K}_{\left[a_{i}\right]}$ que no contienen elementos de $\mathbb{K}_{\left[a_{i+1}\right]}$, se obtiene el siguiente resultado.

Teorema 9 El número de máximos regionales de nivel $a_{i}$ de un complejo cúbico difuso $\mathbb{K}$ es $\beta_{0}\left(\mathbb{K}, a_{i}\right)$.

\section{Aplicaciones}

Las imágenes digitales binarias tienen un papel muy importante en la clasificación y reconocimiento de patrones en imágenes digitales. En [11] se encuentra una excelente introducción a diversos conceptos que se han aplicado para estudiar propiedades topológicas de las imágenes digitales binarias, ya sean bidimensionales o tridimensionales, utilizando elementos de matemáticas discretas. Por ejemplo, para el caso bidimensional, una imagen digital binaria es una tétrada $\left(\mathbb{Z}^{2}, B, m, n\right)$ en la que $B \subset \mathbb{Z}^{2}$ es el conjunto de puntos negros, $\mathbb{Z}^{2}-B$ es el conjunto de puntos blancos y $\{m, n\}=\{4,8\} ; m$ indica el tipo de adyacencia entre dos puntos negros y $n$ el tipo de adyacencia entre dos puntos blancos o entre un punto negro y uno blanco $[23,21,11]$. Utilizando estas adyacencias se definen diversas propiedades topológicas de una imagen digital binaria, tales como sus componentes ( $m$-componentes del conjunto de puntos negros), sus agujeros ( $n$-componentes finitas del conjunto de puntos blancos) y su árbol de adyacencias. Estas propiedades son muy importantes en las tareas de clasificación y reconocimiento de patrones y pueden determinarse mediante diferentes tipos de algoritmos.

A fin de aplicar métodos de la topología algebraica, al conjunto de puntos negros de una imagen binaria se le puede asociar un complejo cúbico [1, 3, 4, $21]$, que en el caso $(m, n)=(8,4)$ es de tipo cerrado, mientras que en el caso $(m, n)=(4,8)$ es abierto [21]. Para ello, a cada $p=\left(x_{1}, x_{2}\right) \in \mathbb{Z}^{2}$ se le asocia el complejo cúbico formado por las celdas que son caras de la 2-celda $s\left(x_{1}, x_{2} ;\{1,2\}\right)$. A este complejo se le llama realización geométrica del punto $p$ y se denota por $|p|$. En este caso, la realización geométrica cerrada de un conjunto $B \subset \mathbb{Z}^{2}$, denotada por $|B|$, es el complejo cúbico cerrado formado por la unión de las realizaciones geométricas de los elementos de $B$, mientras que su realización geométrica abierta, denotada por $|B|^{\circ}$, es el complejo cúbico abierto dado por el interior de la realización geométrica cerrada. La Figura 2 a muestra un subconjunto $B$ formado por 4 puntos de $\mathbb{Z}^{2}$, mientras que en las Figuras $2 \mathrm{~b}$ y $2 \mathrm{c}$ se muestra la realización poliédrica de su realización geométrica cerrada y abierta, respectivamente. 


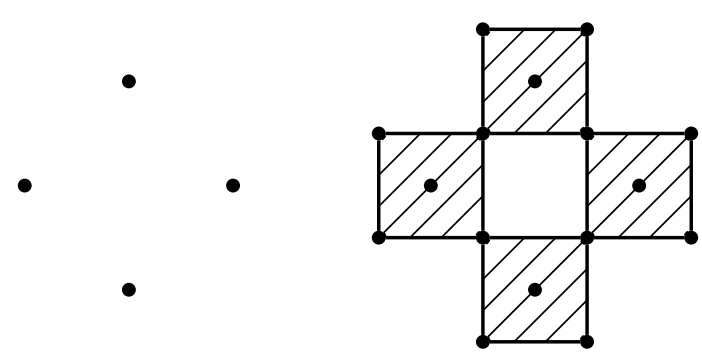

(a)

(b)

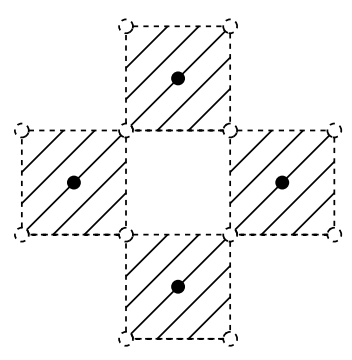

(c)

Figura 2: Realizaciones cerrada y abierta de un subconjunto de $\mathbb{Z}^{2}$.

Hay una relación estrecha entre las propiedades del conjunto $B$ y las de su realización geométrica. Por ejemplo, se puede demostrar que $B$ es 8-conexo si, y sólo si, su realización geométrica cerrada es un complejo cúbico conexo. De igual forma, $B$ es 4-conexo si, y sólo si, su realización geométrica abierta es un complejo cúbico conexo. A partir de estos resultados se puede probar que hay una biyección entre las componentes de una imagen binaria y las componentes de su realización geométrica. Lo mismo ocurre con los agujeros. Además, sus árboles de adyacencias son isomorfos. Por esto, se pueden utilizar los rangos de los grupos de homología del complejo cúbico resultante para determinar el número de componentes y el de agujeros $[3,9,18]$. También se utilizan estos grupos de homología para definir la característica de Euler de una imagen binaria. Un análisis similar se realiza en el caso tridimensional [5].

Para el estudio de las imágenes digitales en tonos de gris se han extendido algunos de los conceptos anteriores utilizando conjuntos difusos [24, 25]. Para este tipo de imágenes también se han definido diversas características topológicas, como sus extremos regionales y sus árboles de componentes [7, 19, 17]. Sin embargo, en la literatura no hay aplicaciones de la matemática difusa a este tipo de imágenes mediante herramientas de la topología difusa. Para ilustrar el uso de la teoría desarrollada en este artículo, se muestra cómo se puede asociar a una imagen en tonos de gris un complejo cúbico difuso y cómo se relacionan las propiedades topológicas de la imagen con las del complejo cúbico difuso resultante.

Definición 14 Una imagen digital bidimensional en tonos de gris es una tétrada $P=\left(\mathbb{Z}^{2}, F, k, l\right)$ en la que $F$ es un subconjunto difuso de $\mathbb{Z}^{2}$ cuyo soporte es finito y $\{k, l\}=\{4,8\}$. El rango de $F$ se representa por $\operatorname{rank}(F)=\left\{a_{0}=\right.$ $\left.0, a_{1}, \ldots, a_{t}\right\}$ 
Una imagen binaria es un tipo particular de imagen en tonos de gris, en la que $\operatorname{rank}(F)=\{0,1\}, F(p)=0$ si $p$ es un punto blanco y $F(p)=1$ si $p$ es un punto negro.

A diferencia del caso binario, los valores de $k$ y $l$ se utilizan para definir los máximos regionales y los mínimos regionales.

Definición 15 Un máximo regional de nivel $a_{i}>0$ de una imagen en tonos de gris $P=\left(\mathbb{Z}^{2}, F, k, l\right)$ es una $k$-componente de $F_{\left[a_{i}\right]}$ que no contiene elementos de $F_{\left[a_{i+1}\right]}$. Un mínimo regional de nivel $a_{i}<1$ de esta imagen es una l-componente finita de $F^{\left[a_{i}\right]}$ que no tiene elementos de $F^{\left[a_{i-1}\right]}$.

Estas definiciones generalizan las de componente y agujero de una imagen binaria, respectivamente.

A partir del conjunto difuso $F$ se puede construir un complejo cúbico difuso llamado realización geométrica de la imagen en tonos de gris. La construcción depende del tipo de adyacencia correspondiente.

Definición 16 Dada una imagen en tonos de gris $P=\left(\mathbb{Z}^{2}, F, 8,4\right)$ se construye un complejo cúbico difuso de la siguiente manera. Si $s=\left(x_{1}, x_{2} ; \Lambda\right)$ es una celda en $\left|\mathbb{Z}^{2}\right|$, se define $G(s)$ como:

1. $G(s)=\max \left\{F\left(x_{1}, x_{2}\right), F\left(x_{1}+1, x_{2}\right), F\left(x_{1}, x_{2}+1\right), F\left(x_{1}+1, x_{2}+1\right)\right\}$ si $\Lambda=\emptyset$,

2. $G(s)=\max \left\{F\left(x_{1}, x_{2}\right), F\left(x_{1}, x_{2}+1\right)\right\}$ si $\Lambda=\{1\}$,

3. $G(s)=\max \left\{F\left(x_{1}, x_{2}\right), F\left(x_{1}+1, x_{2}\right)\right\}$ si $\Lambda=\{2\}$,

4. $G(s)=F\left(x_{1}, x_{2}\right)$ si $\Lambda=\{1,2\}$.

A cada celda s con $G(s) \neq 0$ se le asocia una celda difusa $s^{\prime}$ cuyo soporte es $s$ y cuyo grado de pertenencia es $G(s)$. El conjunto de las celdas difusas $s^{\prime}$ construidas de esta forma es un complejo cúbico difuso llamado la realización geométrica de la imagen $P$ y se denota por $|P|$.

Nótese que, de acuerdo con esta definición, $G(s)=F(p)$ para algún $p$ tal que $s \in|p|$.

Definición 17 Dada una imagen en tonos de gris $P=\left(\mathbb{Z}^{2}, F, 4,8\right)$ se construye un complejo cúbico difuso de la siguiente manera. Si $s=\left(x_{1}, x_{2} ; \Lambda\right)$ es una celda en $\left|\mathbb{Z}^{2}\right|$, se define $H(s)$ como: 
1. $H(s)=\min \left\{F\left(x_{1}, x_{2}\right), F\left(x_{1}+1, x_{2}\right), F\left(x_{1}, x_{2}+1\right), F\left(x_{1}+1, x_{2}+1\right)\right\}$ si $\Lambda=\emptyset$,

2. $H(s)=\min \left\{F\left(x_{1}, x_{2}\right), F\left(x_{1}, x_{2}+1\right)\right\}$ si $\Lambda=\{1\}$,

3. $H(s)=\min \left\{F\left(x_{1}, x_{2}\right), F\left(x_{1}+1, x_{2}\right)\right\}$ si $\Lambda=\{2\}$,

4. $H(s)=F\left(x_{1}, x_{2}\right)$ si $\Lambda=\{1,2\}$.

A cada celda $s$ con $H(s) \neq 0$ se le asocia una celda difusa $s^{\prime}$ cuyo soporte es $s$ y cuyo grado de pertenencia es $H(s)$. El conjunto de las celdas difusas $s^{\prime}$ construidas de esta forma es un complejo cúbico difuso llamado la realización geométrica de la imagen $P$ y se denota por $|P|$.

En este caso también se cumple que $H(s)=F(p)$ para algún $p$ tal que $s \in|p|$.

Los siguientes dos teoremas muestran la relación que existe entre las secciones de una imagen en tonos de gris y las de su realización geométrica.

Teorema 10 Sea $P=\left(\mathbb{Z}^{2}, F, 8,4\right)$ una imagen en tonos de gris. Para cada $a \in I$ se cumple

$$
\left|F_{[a]}\right|=|P|_{[a]},
$$

es decir, la realización geométrica cerrada del a-nivel superior cerrado del conjunto difuso $F$ es igual al a-nivel superior cerrado de la realización geométrica de la imagen $P$.

Demostración. Supóngase primero que $a>0$ y que $s$ es una celda de $\left|F_{[a]}\right|$. Existe $p=\left(x_{1}, x_{2}\right) \in \mathbb{Z}^{2}$ tal que $F(p) \geq a$ y $s \in|p|$. Hay 9 posibles casos para $s$ :

$$
\begin{array}{lll}
s\left(x_{1}-1, x_{2}-1 ; \emptyset\right) & s\left(x_{1}, x_{2}-1 ; \emptyset\right) & s\left(x_{1}-1, x_{2} ; \emptyset\right) \\
s\left(x_{1}, x_{2} ; \emptyset\right) & s\left(x_{1}, x_{2}-1 ;\{1\}\right) & s\left(x_{1}, x_{2} ;\{1\}\right) \\
s\left(x_{1}-1, x_{2} ;\{2\}\right) & s\left(x_{1}, x_{2} ;\{2\}\right) & s\left(x_{1}, x_{2} ;\{1,2\}\right) .
\end{array}
$$

En cualquiera de ellos se verifica que $G(s) \geq F(p)$.

Por ejemplo, si $s=s\left(x_{1}-1, x_{2}-1 ; \emptyset\right)$, entonces

$$
\begin{aligned}
G(s) & =\max \left\{F\left(x_{1}-1, x_{2}-1\right), F\left(x_{1}, x_{2}-1\right), F\left(x_{1}-1, x_{2}\right), F\left(x_{1}, x_{2}\right)\right\} \\
& \geq F\left(x_{1}, x_{2}\right) .
\end{aligned}
$$

Por lo tanto, la celda difusa $s^{\prime}$ cuyo soporte es $s$ y cuyo grado es $G(s)$ pertenece a $|P|$ y en consecuencia $s \in|P|_{[a]}$.

Ahora, si $s \in|P|_{[a]}$, entonces $s=\operatorname{supp}\left(s^{\prime}\right)$ para alguna celda difusa $s^{\prime} \in|P|$ con $\operatorname{deg}\left(s^{\prime}\right) \geq a$. De acuerdo con la definición de $|P|, \operatorname{deg}\left(s^{\prime}\right)=F(p)$ para 
algún $p=\left(x_{1}, x_{2}\right) \in \mathbb{Z}^{2}$. Si, por ejemplo, $s=s\left(x_{1}-1, x_{2}-1 ; \emptyset\right)$ entonces $s \in|p| \operatorname{con} F(p) \geq a$, es decir, $s \in\left|F_{[a]}\right|$.

Cuando $a=0$, es claro el resultado.

Teorema 11 Sea $P=\left(\mathbb{Z}^{2}, F, 4,8\right)$ una imagen en tonos de gris. Para cada $a \in I$ se cumple

$$
\left|F^{[a]}\right|=|P|^{[a]} .
$$

Demostración. Si $s$ es una celda en $\left|F^{[a]}\right|$, entonces existe $p=\left(x_{1}, x_{2}\right) \in \mathbb{Z}^{2}$ tal que $F(p) \leq a$ y $s \in|p|$. De forma similar a la demostración del teorema anterior, se prueba que $H(s) \leq F(p)$. En el caso $H(s)=0$, se deduce que $s \in \operatorname{Bk}(|P|)$, mientras que si $H(s)>0$, la celda difusa $s^{\prime}$ con soporte $s$ y grado de pertenencia igual a $H(s)$ está en $|P|$. En ambos casos, se concluye que $s \in|P|^{[a]}$. Ahora, si $s \in|P|^{[a]}$ y $s \notin \mathrm{Bk}(|P|)$, entonces $s=\operatorname{supp}\left(s^{\prime}\right)$ para algún $s^{\prime} \in|P|$ con $\operatorname{deg}\left(s^{\prime}\right) \leq a$. Esto implica, razonando como en la demostración del teorema previo, que $s \in|p|$ para algún $p$ con $F(p) \leq a$, por lo que $s \in\left|F^{[a]}\right|$. En el caso $s \in \mathrm{Bk}(|P|)$, es claro que $s \in\left|F^{[a]}\right|$.

Utilizando los resultados anteriores y el teorema 1 es inmediata la siguiente propiedad de la realización geométrica de una imagen en tonos de gris, de acuerdo con el tipo de adyacencia correspondiente.

Teorema 12 La realización geométrica de una imagen $P=\left(\mathbb{Z}^{2}, F, 8,4\right)$ es un complejo cúbico difuso cerrado.

De forma similar se demuestra el siguiente resultado.

Teorema 13 La realización geométrica de una imagen $P=\left(\mathbb{Z}^{2}, F, 4,8\right)$ es un complejo cúbico difuso abierto.

Los siguientes teoremas muestran la estrecha relación que hay entre los extremos regionales de una imagen y los de su realización geométrica como complejo cúbico difuso.

Teorema 14 Sea $P=\left(\mathbb{Z}^{2}, F, 8,4\right)$ una imagen en tonos de gris y $M \subset \mathbb{Z}^{2}$. El conjunto $M$ es un máximo regional de $P$ si, y sólo si, su realización geométrica cerrada es un máximo regional de la realización geométrica de $P$.

Demostración. Supóngase que $M$ es un máximo regional de nivel $a_{i}$ de $P$. Por definición, $M$ es una 8-componente de $F_{\left[a_{i}\right]}$, con $a_{i}=F(x)$ para algún $x$ en $M$ y, por tanto, su realización geométrica es un subcomplejo conexo de $|P|_{\left[a_{i}\right]}$. Si $s$ es cualquier celda en $|P|_{\left[a_{i}\right]}$ tal que $|M| \cup\{s\}$ es conexo, entonces existe 
$p \in \mathbb{Z}^{2}$ tal que $s \in|p|$ y $F(p) \geq a_{i}$. Con esto, $|M \cup\{p\}|=|M| \cup|p|$ también es un complejo cúbico conexo, por lo que $M \cup\{p\}$ es un conjunto 8-conexo contenido en $F_{\left[a_{i}\right]}$. Esto implica que $p \in M$ y por tanto $s \in|M|$. Así, $|M|$ es una componente conexa de $|P|_{\left[a_{i}\right]}$.

Ahora, si $|M|$ contiene algún elemento de $|P|_{\left[a_{i+1}\right]}$, entonces existe una celda $s$ tal que $s \in|p|$ para algún $p \in M$ y $s$ es el soporte de una celda difusa $s^{\prime}$ en $|P|$ con $\operatorname{deg}\left(s^{\prime}\right) \geq a_{i+1}$. Como $\operatorname{deg}\left(s^{\prime}\right)=F(q)$ para algún $q$ tal que $s \in|q|$, el complejo cúbico $|M| \cup|q|$ es conexo, lo que implica que $M \cup\{q\}$ es 8-conexo y por tanto $q \in M$, con $F(q)=\operatorname{deg}\left(s^{\prime}\right) \geq a_{i+1}$, contradiciendo que $M$ es un máximo regional de nivel $a_{i}$.

Supóngase ahora que $|M|$ es un máximo regional de nivel $a_{i}$ de $|P|$. Como $|M|$ es conexo, $M$ es 8 -conexo. Además, para cada $p \in M$, la 2 -celda $s(p,\{1,2\})$ es el soporte de una celda difusa $s^{\prime}$ en $|P|$, con grado de pertenencia $G(s)=$ $F(p) \geq a_{i}$. Así, $M \subset F_{\left[a_{i}\right]}$. Si $p$ es 8-adyacente a $M$ у $p \in F_{\left[a_{i}\right]}$, entonces $|M| \cup|p|$ es un complejo cúbico conexo contenido en $|P|_{\left[a_{i}\right]}$, por lo que $|p| \subset|M|$. Por tanto, $p \in M$ y $M$ es una 8-componente de $F_{\left[a_{i}\right]}$. Además, si $M$ contiene algún elemento $p$ de $F_{\left[a_{i+1}\right]}$ entonces la celda $s=s\left(x_{1}, x_{2} ;\{1,2\}\right)$ es el soporte de una celda difusa $s^{\prime} \operatorname{de}|P| \operatorname{con} \operatorname{deg}\left(s^{\prime}\right) \geq a_{i+1}$ y $s \in|M|$, contradiciendo que $|M|$ es un máximo regional de nivel $a_{i}$ de $|P|$.

Como consecuencia de este teorema se tiene el siguiente resultado.

Teorema 15 El árbol superior de una imagen en tonos de gris $P=\left(\mathbb{Z}^{2}, F, 8,4\right)$ es isomorfo al árbol superior de su realización geométrica.

Un resultado similar se obtiene en el caso de las imágenes de tipo $(4,8)$ para sus mínimos regionales.

Teorema 16 Sea $P=\left(\mathbb{Z}^{2}, F, 4,8\right)$ una imagen en tonos de gris y $M$ un subconjunto finito de $\mathbb{Z}^{2}$. El conjunto $M$ es un mínimo regional de $P$ si, y sólo si, su realización geométrica cerrada es un mínimo regional de la realización geométrica de $P$.

\section{Conclusiones y trabajo futuro}

En este artículo se ha mostrado cómo se puede generalizar el concepto de complejo cúbico utilizando elementos de matemática difusa y se han demostrado algunas de sus propiedades básicas. El teorema 2 permite concluir que la generalización propuesta es una buena extensión del concepto de complejo cúbico 
en el sentido propuesto por Lowen. El lema 2 y el teorema 4 muestran que el concepto de máximo regional propuesto generaliza el de componente conexa.

Aunque los grupos de homología propuestos para un complejo cúbico difuso están definidos a partir de grupos de homología singular de pares de espacios topológicos, en el caso de los complejos cerrados o abiertos es posible estudiar sus propiedades a partir de la teoría de los complejos cúbicos ordinarios. A diferencia de otras propuestas para definir grupos de homología asociados a objetos de la matemática difusa, el teorema 9 muestra que el rango del 0-grupo de homología definido en este artículo se relaciona de manera cuantitativa con una propiedad de conexidad del complejo cúbico difuso correspondiente.

Las definiciones 16 y 17 muestran cómo se puede construir un complejo cúbico difuso a partir de una imagen en tonos de gris, dependiendo del tipo de adyacencia empleado. Los teoremas 14, 15 y 16 muestran que el complejo cúbico difuso correspondiente tiene propiedades topológicas similares a la de la imagen digital correspondiente. Puesto que las secciones de este complejo cúbico difuso son complejos cúbicos ordinarios, esto permitirá aplicar herramientas de la topología algebraica al estudio de las imágenes en tonos de gris.

Como trabajo futuro se plantea buscar métodos que faciliten el cálculo del rango de los grupos de homología de un complejo cúbico difuso mediante transformaciones homotópicas. También se plantea investigar el significado del rango de los grupos de homología de órdenes superiores de estos complejos cúbicos difusos y sus implicaciones en el estudio de las imágenes en tonos de gris. De igual manera, se plantea investigar la existencia de invariantes topológicos similares a la característica de Euler para esta clase de complejos cúbicos y determinar su utilidad en el estudio de las imágenes digitales en tonos de gris. Por último, se pueden investigar las aplicaciones de la teoría desarrollada en el análisis de imágenes digitales en dimensiones superiores.

\section{Referencias}

[1] Allili, M.; Mischaikow, K.; Tannenbaum, A. (2001) "Cubical homology and the topological classification of 2D and 3D imagery", en: Proceedings of the International Conference on Image Processing 2001, Vol. 2, IEEE, Grecia: 173-176.

[2] Bairamov, S.; Gunduz, C. (2010) "The Čech homology theory in the category of Šstak fuzzy topological spaces", International Journal of Contemporary Mathematical Sciences 5(9): 433-448. 
[3] Bykov, A.I.; Zerkalov, L.G. (1996) "Algorithms for homotopy classification of binary images", Pattern Recognition 29(4): 565-574.

[4] Bykov, A.I.; Zerkalov, L.G.; Albores Velasco, F.J.; Rodríguez Pineda, M. A. (1998) "New connected components algorithm and invariant transformations of digital images", Pattern Recognition 31(8): 1089-1098.

[5] Bykov, A.I.; Zerkalov, L.G.; Rodríguez Pineda, M.A. (1999) "Index of a point of 3-D digital binary image and algorithm for computing its Euler characteristic", Pattern Recognition 32(5): 845-850.

[6] Chang, C.L. (1968) "Fuzzy topological spaces", Journal of Mathematical Analysis and Applications 24(1): 182-190.

[7] Couprie, M.; Nivando Bezerra, F.; Bertrand, G. (1999) "Grayscale image processing using topological operators", Proceedings SPIE Vision Geometry VIII 3811: 261-272.

[8] Cuvalcioglu, G.; Citil, M. (2006) "On fuzzy homotopy theory”, Advanced Studies in Contemporary Mathematics 12(1): 163-166.

[9] Kaczynski, T.; Mischaikow, K.; Mrozek, M. (2003) "Computing homology”, Homology, Homotopy and Applications 5(2): 233-256.

[10] Kerre, E.E. (2005) "A historical overview of fuzzy mathematics", New Mathematics and Natural Computation 1(1): 1-26.

[11] Kong, T.Y.; Rosenfeld, A. (1989) "Digital topology: introduction and survey”, Computer Vision, Graphics and Image Processing 48: 357-393.

[12] Li, S.G. (2000) "Connectedness in L-fuzzy topological spaces", Fuzzy Sets and Systems 116(3): 361-368.

[13] Lowen, R. (1978) "A comparison of different compactness notions in fuzzy topological spaces", Journal of Mathematical Analysis and Applications 64: 446-454.

[14] Lowen, R. (1980) “Convex fuzzy sets”, Fuzzy Sets and Systems 3(3): 291310.

[15] Lowen, R. (1981) "Connectedness in fuzzy topological spaces", Rocky Mountain Journal of Mathematics 11(3): 427-433.

[16] Lubczonok, P. (1990) "Fuzzy vector spaces", Fuzzy Sets and Systems 38(3): 329-343. 
[17] Monasse, P.; Guichard, F. (2000) "Fast computation of a contrast-invariant image representation", IEEE Transactions on Image Processing 9(5): 860-872.

[18] Mrozek, M.; Batko, B. (2009) "Coreduction homology algorithm", Discrete Computational Geometry 41: 96-118.

[19] Najman, L.; Couprie, M. (2006) "Building the component tree in quasilinear time", IEEE Transactions on Image Processing 15(11): 3531-3539.

[20] Niethammer, M.; Kalies, W.D.; Mischaikow, K.; Tannenbaum, A. (2006) "On the detection of simple points in higher dimensions using cubical homology”, IEEE Transactions on Image Processing 15(8): 2462-2469.

[21] Rodríguez-Pineda, M.A. (1997) El Concepto de Índice de un Punto Lattice en el Análisis Homotópico de 2D y 3D Imágenes y su Realización Algorítmica. Tesis de doctorado, Benemérita Universidad Autónoma de Puebla, México.

[22] Rosenfeld, A. (1971) "Fuzzy groups", Journal of Mathematical Analysis and Applications 35(3): 512-517.

[23] Rosenfeld, A. (1979) "Digital topology", The American Mathematical Monthly 86(8): 621-630.

[24] Rosenfeld, A. (1979) "Fuzzy digital topology", Information and Control 40(1): 76-87.

[25] Rosenfeld, A. (1983) "On connectivity properties of grayscale pictures", Pattern Recognition 6(1): 47-50.

[26] Salleh, A.R. (1993) "The homotopy property of the induced homomorphisms on homology groups of fuzzy topological spaces", Fuzzy Sets and Systems 56(1): 111-116.

[27] Sidky, F.I.; Mishref, M.A. (1991) "Fuzzy cosets and cyclic and abelian fuzzy subgroups", Fuzzy Sets and Systems 43(2): 243-250.

[28] Wang-Jin, L.; Chong-You, Z. (1985) "Singular homology groups of fuzzy topological spaces", Fuzzy Sets and Systems 15(2): 199-207.

[29] Ying-Ming, L.; Mao-Kang, L. (1996) Fuzzy Topology. World Scientific, Singapore. 
[30] Zadeh, L.A. (1965) "Fuzzy sets”, Information and Control 8(3): 238-253.

[31] Zadeh, L.A.; Fu, K. S.; Tanaka, K.; Shimura, M. (1975) Fuzzy Sets and Their Applications to Cognitive and Decision Processes. Academic Press, New York.

[32] Ziou, D.; Allili, M. (2002) "Generating cubical complexes from image data and computation of the Euler number", Pattern Recognition 35(12): 2833-2839. 
